\title{
REFERENCES
}

1. G. Polya, On the mean-value theorem corresponding to a given linear homogeneous differential equation, Trans. Amer. Math. Soc. 24 (1922), 312-324.

2. E. F. Beckenbach and R. Bellman, Inequalities, Springer, Berlin, 1961.

3. F. R. Gantmacher and M. G. Krein, Oszillationsmatrizen, Oszillationskerne und kleine Schwingungen mechanischer Systeme, Akademie-Verlag, Berlin, 1960.

RAND Corporation, Santa Monica

\section{A VOLTERRA EQUATION WITH A VERY SINGULAR KERNEL ${ }^{1}$}

E. L. ROETMAN

For continuous, or mildly singular kernels, the Volterra equations of second kind can be solved with a Neumann series which converges for the entire complex $\lambda$ plane. The purpose of the present note is to show, by studying a special case, what can happen if the kernel is very singular. In particular, we find that the equation can have finite spectral values, in fact the circle $|\lambda|=1$ can be a natural boundary for the resolvent kernel, and that it is not easy to describe a "natural" domain and range for the operator in terms of the usual classes of functions.

Consider the Volterra type integral equation for complex valued functions of a real variable given by

$$
\phi=a+\lambda k * \phi
$$

where

$$
k(t)=\left(i / \pi^{1 / 2}\right) t^{-3 / 2} \exp (i \pi / 4+i / t)
$$

and * denotes the convolution,

$$
f * g=\int_{\theta}^{t} f(t-s) g(s) d s .
$$

(The study of the boundary value problem for the equation of vibration of elastic bars leads to the related equation

Received by the editors November 13, 1964.

${ }^{1}$ Based in part on the author's thesis, written under the guidance of Professor W. Fulks, submitted to Oregon State University. 


$$
\phi=a+\lambda k * \Phi
$$

which is solved in [1] and which does not exhibit the pathologies discussed here.)

By formal substitution we obtain the Neumann series

$$
\phi=a+\lambda k * a+\lambda^{2} k *(k * a)+\cdots .
$$

It has been proved in $[1$, Appendix I] that the integral $k * a$ exists for $0 \leqq t \leqq T$ if $a$ is $\operatorname{CBV}[0, T],{ }^{2}$ that for this class of functions

$$
k *(k * a)=(k * k) * a \equiv k_{2} * a,
$$

and that

$$
k_{2}(t)=\left(2 i / \pi^{1 / 2}\right) t^{-3 / 2} \exp (i \pi / 4+4 i / t) .
$$

Since $k_{2}(t)$ has the same order singularity as $k(t)$, the above results assure that $k_{2} * a$ exists and

$$
k *(k *(k * a))=\left(k * k_{2}\right) * a \equiv k_{3} * a .
$$

$k_{3}$ can also be evaluated as above; in fact, by [1, Appendix I], (3) can be written

$$
\phi=a+\sum_{n=1}^{\infty} \lambda^{n} k_{n} * a
$$

where

$$
k_{n}(t)=\left(n i / \pi^{1 / 2}\right) t^{-3 / 2} \exp \left(i \pi / 4+i n^{2} / t\right)
$$

We prove

THEOREM 1. If $a(t)$ is $C B V[0, T]$ and $|\lambda|<1$, then (4) defines a continuous solution of (1).

By integration by parts

$$
\begin{aligned}
& \int_{0}^{t} k_{n}(t-s) a(s) d s=n \exp (i \pi / 4) \pi^{1 / 2}\left\{\left(i / n^{2}\right) \exp \left(i n^{2} / t\right) t^{1 / 2} a(0)\right. \\
& \left.+\left(i / n^{2}\right) \int_{0}^{t} \exp \left(i n^{2} /(t-s)\right)\left[-1 / 2(t-s)^{-1 / 2} a(s) d s+(t-s)^{1 / 2} d a(s)\right]\right\} .
\end{aligned}
$$

Thus with $A=\max _{[0, T]} a(t)$

$$
\begin{aligned}
\left|k_{n} * a\right| & \leqq\left(1 /\left(n \pi^{1 / 2}\right)\right) \\
\cdot & \left\{A T^{1 / 2}+A \int_{0}^{t} \frac{1}{2}(t-s)^{-1 / 2} d s+\int_{0}^{t}(t-s)^{1 / 2} d V(a ; 0, s)\right\},
\end{aligned}
$$

$2 \mathrm{CBV}[0, T]$ means that class of functions which are continuous and of bounded variation on $[0, T]$. 
where $V(a ; 0, s)$ is the total variation of $a$ from 0 to $s$. Hence

$$
\left|k_{n} * a\right| \leqq(1 / n)(2 A+V(a ; 0, T))(T / \pi)^{1 / 2}=B / n
$$

where $B$ is independent of $t$ and $n$. Therefore

$$
\left|\sum_{n=1}^{\infty} \lambda^{n} k_{n} * a\right| \leqq B \sum_{n=1}^{\infty}|\lambda|^{n} / n
$$

so that (4) converges uniformly and absolutely on $[0, T]$.

We must yet show that $\phi$ defined by (4) is continuous and that (1) is satisfied. Suppose $h>0$; we have

$$
\begin{aligned}
D= & \int_{0}^{t+h} s^{-3 / 2} \exp \left(i n^{2} / s\right) a(t+h-s) d s \\
& -\int_{0}^{t} s^{-3 / 2} \exp \left(i n^{2} / s\right) a(t-s) d s \\
= & \int_{0}^{t} s^{-3 / 2} \exp \left(i n^{2} / s\right)[a(t+h-s)-a(t-s)] d s \\
& +\int_{t}^{t+h} s^{-3 / 2} \exp \left(i n^{2} / s\right) a(t+h-s) d s .
\end{aligned}
$$

By a mean value theorem for BV functions [2, p. 623],

$$
\begin{aligned}
|D| \leqq & \{|a(t+h)-a(t)|+V(a(s) ; t, t+h)+V(a(s) ; 0, h)\} M_{1} \\
& +\{|a(h)|+V(a(s) ; 0, h)\} M_{2}
\end{aligned}
$$

where

$$
M_{1}=\sup _{0 \leqq t_{1}<t_{1} \leqq T}\left|\int_{t_{1}}^{t_{2}} s^{-8 / 2} \exp \left(i n^{2} / s\right) d s\right|
$$

and

$$
M_{2}=\sup _{t \leqq t_{1}<t_{2} \leqq t_{+h}}\left|\int_{t_{1}}^{t_{2}} s^{-3 / 2} \exp \left(i n^{2} / s\right) d s\right| .
$$

Now, $a$ and $V(a)$ are continuous on $[0, T]$ and, since the integral exists, $M_{2}=o(1)$. Thus $|D| \rightarrow 0$ as $h \rightarrow 0$. For $h<0$, we have the corresponding result so that each term in (4) is continuous. This implies, with the uniform convergence, that $\phi$ is continuous.

Also, 


$$
\begin{aligned}
\lambda k * \phi & =\lambda k * a+\lambda k *\left(\sum \lambda^{n} k_{n} * a\right) \\
& =\sum \lambda^{n} k_{n} * a=-a+\phi
\end{aligned}
$$

so that (4) is a solution of (1).

Using (6), it is not difficult to show that, for $|\lambda|<1$, (4) can be written

$$
\phi=a+\int_{0}^{t} R(\lambda ; t-s) a(s) d s
$$

where

$$
R(\lambda ; t)=\left(1 / \pi^{1 / 2}\right) e^{i \pi / 4} t^{-3 / 2} \sum_{n=1}^{\infty} n \lambda^{n} \exp \left(i n^{2} / t\right)
$$

By [3, Theorem 8, p. 424], the circle $|\lambda|=1$ is a natural boundary of $R(\lambda, t)$.

To further illustrate the behavior of this equation, we give an elementary proof that $\lambda=1$ is a spectral value of the equation. We assume that $\lambda=1$ and $a \equiv 1$ so that (4) gives

$$
\phi(t)=1+\sum_{n=1}^{\infty} \int_{0}^{t} k_{n}(s) d s .
$$

We let $u=s^{-1}$ and integrate by parts twice to obtain

$$
\int_{0}^{t} k_{n}(s) d s=C t^{1 / 2} n^{-1} \exp \left(i n^{2} / t\right)+O\left(n^{-3}\right)
$$

uniformly in $n$. Set $t=1 / 2 \pi m$ where $m$ is any positive integer. Then

$$
\int_{0}^{t} k_{n}(s) d s=C \frac{1}{(2 \pi m)^{1 / 2}} \frac{1}{n}+O\left(n^{-3}\right),
$$

and

$$
\phi\left(\frac{1}{2 \pi m}\right)=1+C^{\prime} \sum n^{-1}+\sum O\left(n^{-3}\right) .
$$

Since the second series converges and the first diverges, we see that the series (8) fails to define $\phi(t)$ for $t=1 / 2 \pi m$.

On the other hand, we can show that: if $\lambda=e^{i \theta}$ and $a(t)$ is differentiable with $a^{\prime} \in C B V[0, T]$ and $a(0)=0$, then (4) defines a continuous solution of (1).

With the above hypothesis, (6) can be written 


$$
\begin{aligned}
k_{n} * a=C n^{-3} & \left\{\exp \left(\frac{i n^{2}}{t}\right)\left[-\frac{1}{2} t^{3 / 2} a(t)+t^{5 / 2} a^{\prime}(t)\right]\right. \\
& +\int_{0}^{t} \exp \left(\frac{i n^{2}}{t-s}\right)\left[\frac{3}{4}(t-s)^{1 / 2} a(s) d s\right. \\
& \left.\left.-3(t-s)^{3 / 2} a^{\prime}(s) d s+(t-s)^{5 / 2} d a^{\prime}(s)\right]\right\} .
\end{aligned}
$$

As in the proof of Theorem 1 , we obtain $\left|k_{n} * a\right| \leqq B n^{-3}$ where $B$ is independent of $n$ and $t$. Thus the series (4) converges uniformly and absolutely so that the conclusions follow exactly as in Theorem 1.

\section{ReferenCes}

1. E. L. Roetman, Vibration of elastic bars, Ph.D. Thesis, Oregon State University, Corvallis, Ore., 1963.

2. E. W. Hobson, Theory of functions of a real variable, Vol. 1, Dover, New York, 1957.

3. R. Cooper, The behavior of certain series associated with limiting cases of elliptic theta-functions, Proc. London Math. Soc. (2) 27 (1928), 410-426.

STEVENS Institute of TechNology 